\title{
Review Article \\ Phytochemical and Pharmacological Studies on the Genus Psoralea: A Mini Review
}

\author{
Cong-Cong Li, ${ }^{1}$ Teng-Long Wang, ${ }^{1}$ Zhong-Qun Zhang, ${ }^{1}$ Wen-Qiang Yang, ${ }^{2}$ Yue-Fei Wang, \\ Xin Chai, ${ }^{1}$ Chun-Hua Wang, ${ }^{1,3}$ and Zheng $\mathrm{Li}^{3}$ \\ ${ }^{1}$ Tianjin Key Laboratory of Modern Chinese Medicine, Tianjin University of Traditional Chinese Medicine, \\ Tianjin 300193, China \\ ${ }^{2}$ College of Pharmacy, Linyi University, Linyi 276000, China \\ ${ }^{3}$ College of Pharmaceutical Engineering of Traditional Chinese Medicine, Tianjin University of Traditional Chinese Medicine, \\ Tianjin 300193, China
}

Correspondence should be addressed to Xin Chai; chaixinphd@aliyun.com and Chun-Hua Wang; pharmwch@126.com

Received 9 September 2016; Revised 9 October 2016; Accepted 17 October 2016

Academic Editor: Wenyi Kang

Copyright (C) 2016 Cong-Cong Li et al. This is an open access article distributed under the Creative Commons Attribution License, which permits unrestricted use, distribution, and reproduction in any medium, provided the original work is properly cited.

\begin{abstract}
The genus Psoralea, which belongs to the family Fabaceae, comprises ca. 130 species distributed all over the world, and some of the plants are used as folk medicine to treat various diseases. Psoralea corylifolia is a typical example, whose seeds have been widely used in many traditional Chinese medicine formulas for the treatment of various diseases such as leucoderma and other skin diseases, cardiovascular diseases, nephritis, osteoporosis, and cancer. So, the chemical and pharmacological studies on this genus were performed in the past decades. Here, we give a mini review on this genus about its phytochemical and pharmacological studies from 1910 to 2015 .
\end{abstract}

\section{Introduction}

The genus Psoralea, which belongs to the family Fabaceae, comprises ca. 130 species mainly distributed in South Africa, North and South America, and Australia, a few of which are native to Asia and temperate Europe [1]. Among them, several species have been widely used as herbal medicine in China, India, and other countries. Modern pharmacological researches show that the plants in Psoralea genus have antimicrobial, antipregnancy, estrogenic, antitumor, antioxidant, and many other pharmacological activities $[1,2]$. For example, P. corylifolia is the sole species of the genus distributing in China, and its seeds are used as a famous traditional Chinese medicine (TCM), having the effects of kidney impotence and warming spleen and stopping diarrhea and included by Pharmacopoeia of People's Republic of China [3]. Here, we review the progress achieved in phytochemical studies on the genus Psoralea, list the compounds isolated from this genus over the past decades, and introduce the biological activities of these ingredients.

\section{Phytochemistry}

To the best of our knowledge, the first phytochemical investigation on the genus Psoralea can be traced back to 1910 [4]. In 1933, Jois and his coworkers obtained the first pure compound called psoralen (51) from P. corylifolia [4]. Up to 2015, the total number of identified secondary metabolites from the genus Psoralea amounts to 129, including flavonoids, coumarins, phenols, benzofurans, benzopyrans, quinines, sesquiterpenoids, triterpenoids, steroids, and some other components. The structures of these compounds are shown in Figure 1. Their names and the corresponding plant sources are compiled in Table 1.

2.1. Flavonoids. Previous chemical investigations have indicated that flavonoids were the most frequently occurring constituents of the genus Psoralea. Fifty flavonoids, 1 50, have been isolated and elucidated from the genus Psoralea, most of which were isolated from $P$. corylifolia, while isovitexin (2) was got from P. plicata [5]. Various types of 

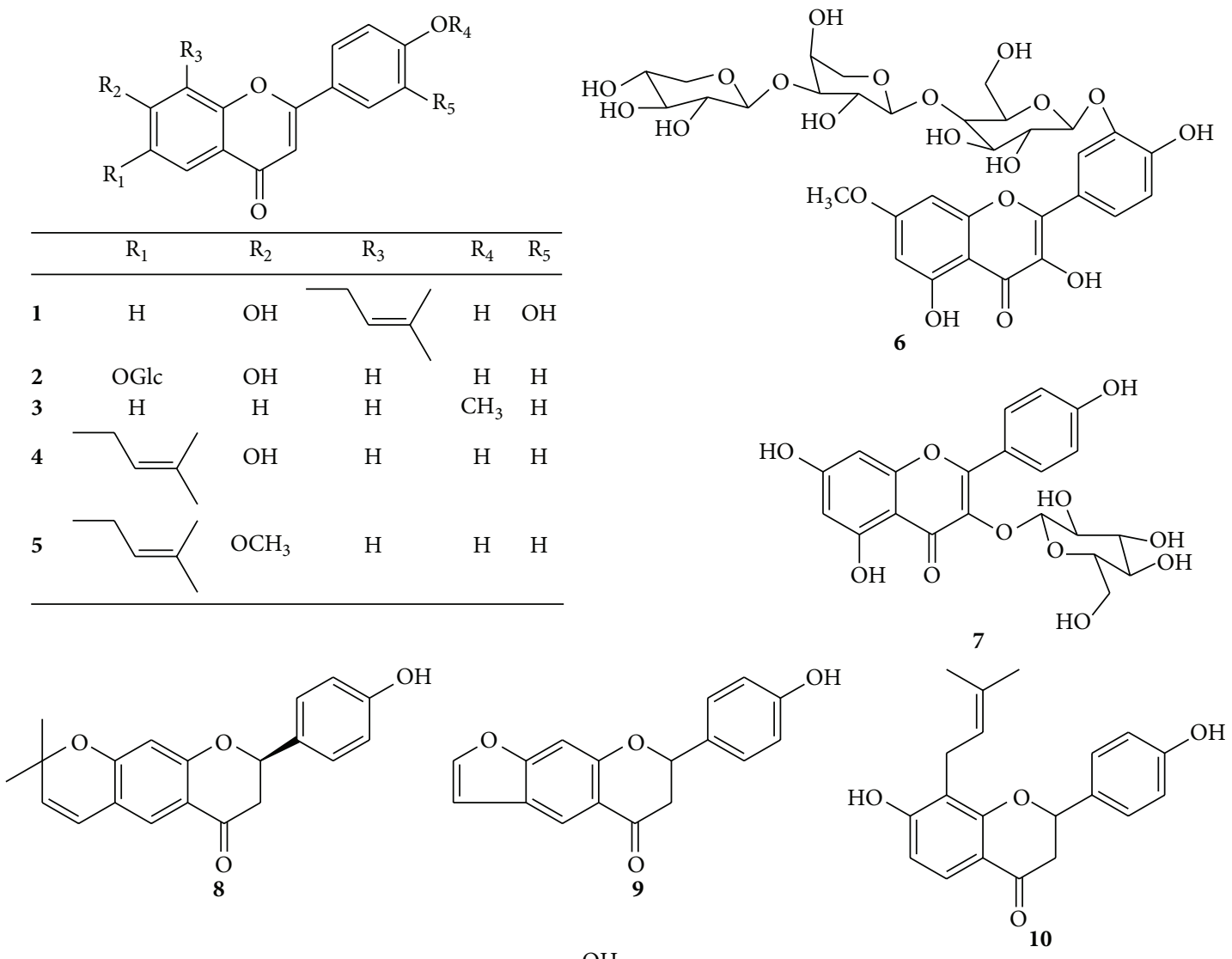

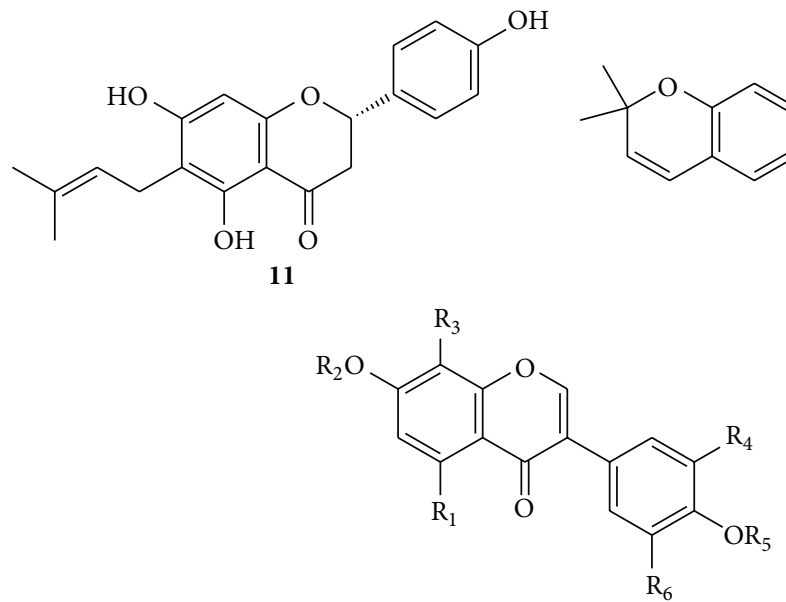<smiles>O=c1c(OC(O)OC(CO)C(O)C(O)CO)c(-c2ccc(O)cc2)oc2cc(O)cc(O)c12</smiles>

\begin{tabular}{ccccccc}
\hline & $\mathrm{R}_{1}$ & $\mathrm{R}_{2}$ & $\mathrm{R}_{3}$ & $\mathrm{R}_{4}$ & $\mathrm{R}_{5}$ & $\mathrm{R}_{6}$ \\
\hline $\mathbf{1 3}$ & $\mathrm{OH}$ & $\mathrm{H}$ & $\mathrm{H}$ & $\mathrm{H}$ & $\mathrm{H}$ & $\mathrm{H}$ \\
$\mathbf{1 4}$ & $\mathrm{H}$ & $\mathrm{H}$ & $\mathrm{H}$ & $\mathrm{H}$ & $\mathrm{H}$ & $\mathrm{H}$ \\
$\mathbf{1 5}$ & $\mathrm{H}$ & $\mathrm{H}$ & $\mathrm{H}$ & $\mathrm{H}$ & $\mathrm{H}$ & $\mathrm{H}$ \\
$\mathbf{1 6}$ & $\mathrm{H}$ & $\mathrm{H}$ & $\mathrm{H}$ & $\mathrm{CHO}$ & $\mathrm{H}$ & $\mathrm{H}$ \\
$\mathbf{1 7}$ & $\mathrm{OH}$ & $\mathrm{H}$ & $\mathrm{H}$ & $\mathrm{H}$ & $\mathrm{CH}_{3}$ & $\mathrm{H}$ \\
$\mathbf{1 8}$ & $\mathrm{H}$ & $\mathrm{CH}_{3}$ & $\mathrm{H}$ & $\mathrm{CHO}$ & $\mathrm{H}$ & $\mathrm{H}$ \\
$\mathbf{1 9}$ & $\mathrm{H}$ & $\mathrm{H}$ & $\mathrm{H}$ & $\mathrm{H}$ & $\mathrm{H}$ \\
& & & & $\mathrm{H}$ & $\mathrm{H}$ & $\mathrm{H}$ \\
$\mathbf{2 0}$ & $\mathrm{H}$ & $\mathrm{CH}$ & $\mathrm{H}$ & $\mathrm{H}$ & $\mathrm{H}$ & $\mathrm{H}$ \\
$\mathbf{2 1}$ & $\mathrm{H}$ & $\mathrm{Glc}$ & $\mathrm{H}$ & $\mathrm{H}$ & $\mathrm{H}$ & $\mathrm{H}(6 \rightarrow 1)-\mathrm{Rha}$ \\
$\mathbf{2 2}$ & $\mathrm{H}$ & $\mathrm{H}$ & $\mathrm{H}$ & & $\mathrm{H}$ \\
\hline
\end{tabular}

(a)

Figure 1: Continued. 
<smiles>COc1ccc2c(=O)c(-c3cccc4c3C=COC4)coc2c1</smiles>

23<smiles>CC1(C)C=Cc2cc(-c3coc4cc(O)ccc4c3=O)ccc2O1</smiles>

24<smiles>CC1(C)CCc2cc(-c3coc4cc(O)ccc4c3=O)ccc2O1</smiles>

27<smiles>CC(C)=CCC/C(C)=C/Cc1cc(-c2coc3cc(O)ccc3c2=O)ccc1O</smiles>

25

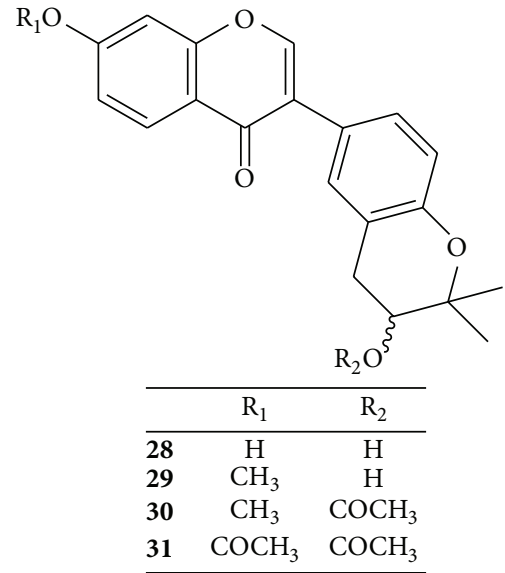<smiles>CC(C)=CCCC1(C)C=Cc2ccc(-c3coc4cc(O)ccc4c3=O)cc2O1</smiles>

26

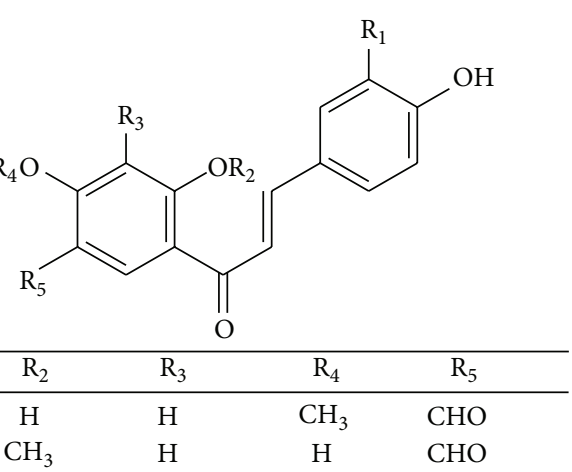

$\mathrm{CHO}$

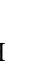<smiles>CCC=C(C)C</smiles>

$34 \quad \mathrm{H}$<smiles>CC(C)(O)C1CC2C(O)=C(C(=O)/C=C/c3ccc(O)cc3)C=CC2O1</smiles>

$\begin{array}{lllll}35 & \mathrm{H} & \mathrm{H} & \mathrm{H}\end{array}$

$\mathrm{CH}_{3}$

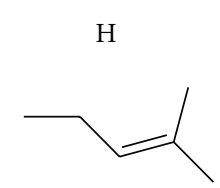

$36 \mathrm{OH} \quad \mathrm{H}$

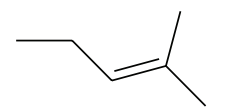

$37 \quad \mathrm{H} \quad \mathrm{H}$

$\mathrm{H}$

$\mathrm{CH}_{3}$

$\mathrm{H}$<smiles>CC(C)(O)[C@H]1Cc2c(ccc(C(=O)/C=C/c3ccc(O)cc3)c2O)O1</smiles><smiles>CC#CC(=O)c1ccc(O)c2c1OC(C)(C)C(O)C2</smiles>

(b)<smiles>C=C(C)C(O)Cc1c(O)ccc(C(=O)/C=C/c2ccc(O)cc2)c1O</smiles><smiles>C=C(C)C(O)Cc1c(O)ccc(C(=O)CC)c1O</smiles>

Continued. 
<smiles>C=C(O)C(O)Cc1c(O)ccc(C(=[18O])/C=C/c2ccc(O)cc2)c1O</smiles><smiles>CC1(C)C=Cc2c(cc(O)c(C(=O)/C=C/c3ccc(O)cc3)c2O)O1</smiles><smiles>CC1(C)C=Cc2cc(C(=O)/C=C/c3ccc(O)cc3)c(O)cc2O1</smiles><smiles>[R3]c1cc([O+])c(CC=C(C)C)cc1C(=O)/C=C/c1ccc(O)c(Br)c1</smiles><smiles>COc1cc(C(=O)/C=C/c2ccc3c(c2)OCO3)c(O)c2ccoc12</smiles>

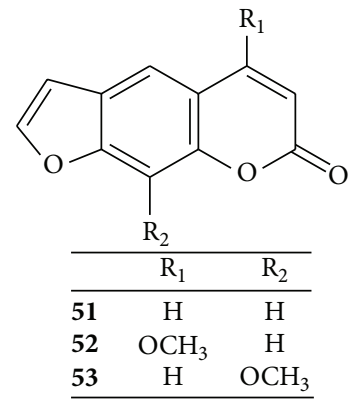<smiles>O=c1ccc2c(ccc3ccoc32)o1</smiles><smiles>O=c1ccc2ccc3occc3c2o1</smiles><smiles>O=c1oc2cc3occc3cc2c2oc3cc(O)ccc3c12</smiles><smiles>CC(C)(O)C1Cc2cc3c(cc2O1)oc1c2ccc(O)cc2oc(=O)c31</smiles><smiles>CC(C)=CCc1cc2c(cc1O)oc(=O)c1c3ccc(O)cc3oc21</smiles><smiles></smiles>

59<smiles>CC1(C)CCc2cc3c(cc2O1)oc(=O)c1c2ccc(O)cc2oc31</smiles><smiles></smiles><smiles>CC1(C)Oc2cc3oc(=O)c4c5ccc(O)cc5oc4c3cc2[C@H](O)[C@@H]1O</smiles>

62<smiles>CC1(C)C=Cc2cc3c(cc2O1)oc1c2cc(O)ccc2oc(=O)c31</smiles>

63

(c)<smiles></smiles>

64

Figure 1: Continued. 
<smiles>CCN(CC)CCOc1c2ccoc2cc2oc(=O)ccc12</smiles><smiles>O=c1oc2ccccc2cc1-c1ccccc1</smiles><smiles>OCc1ccc(O)cc1</smiles><smiles>O=Cc1ccc(O)cc1</smiles><smiles>O=C(O)c1ccc(O)cc1</smiles>

65<smiles>C=CC(C)(/C=C/c1ccc(O)cc1)CCC=C(C)C</smiles>

70<smiles>[R20]C(=O)C=Cc1ccc(O)c(CC=C(C)C)c1</smiles><smiles>C=CC(C)(CC/C=C/c1ccc(O)cc1)C1OC1(C)C</smiles>

74<smiles>C=CC(C)(CC=C(C)C)CC(=C)c1ccc(O)cc1</smiles><smiles>C=C[C@](C)(/C=C/c1ccc(O)cc1)CCC=C(C)C</smiles><smiles>C=C[C@@](C)(CC/C=C/c1ccc(O)cc1)C(O)C(C)(C)O</smiles>
77<smiles>C=Cc1ccc(O)cc1</smiles>

81<smiles>C=C[C@](C)(/C=C/c1ccc(O)cc1)CCC1OC1(C)C</smiles>

82<smiles>C=C[C@]1(C)CCC(C(=C)C)[C@H](c2ccc(O)cc2)C1</smiles>

85

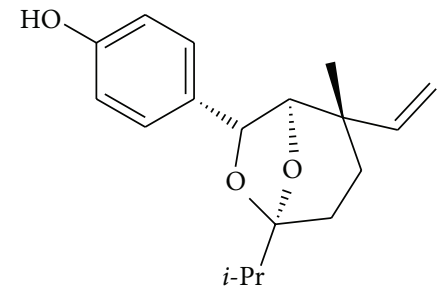

89<smiles>CCOC(=O)CCc1cc2ccoc2cc1O</smiles><smiles>CCOC(=O)CCc1ccc2occc2c1O</smiles>

87

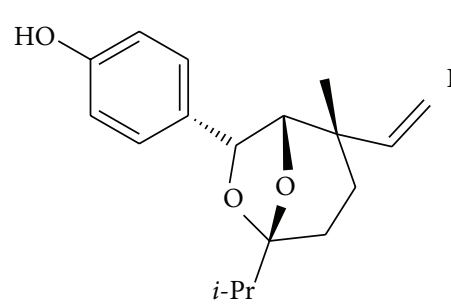

90<smiles>C=C[C@]1(C)CC[C@@H](C(C)(C)O)[C@H](c2ccc(O)cc2)C1</smiles>

83<smiles>C=C[C@]1(C)CC[C@@H](C(=C)C)O[C@H]1[C@H](O)c1ccc(O)cc1</smiles>

88<smiles>C=C[C@]1(C)CC[C@@H](C(=C)C)[C@H](c2ccc(O)cc2)C1</smiles>

84

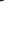

(d) 
<smiles>C=C[C@](C)(CCC=C(C)C)[C@H]1Oc2ccc(/C=C/[C@@](C)(C=C)CCC=C(C)C)cc2O[C@@H]1c1ccc(O)c(O)c1</smiles><smiles>C=C[C@](C)(/C=C/c1ccc(O)cc1)/C=C/c1ccc(OC(C)(C)[C@@H](O)CC[C@](C)(C=C)CCC=C(C)C)cc1</smiles><smiles>Cc1c(C)c2c(c(C)c1O)CC[C@](C)(CCC[C@H](C)CCC[C@H](C)CCCC(C)C)O2</smiles><smiles>CC1=CC(=O)CC(C)(C)[C@@]1(O)/C=C/C(C)(C)OC1OC(O)C(O)C(O)C1O</smiles><smiles>CCOC(=O)CCc1ccc2occc2c1O</smiles>

99<smiles>COC(=O)/C=C\c1ccc2c(c1)C=CC(C)(C)O2</smiles><smiles>COC(=O)C=Cc1ccc2c(c1)C=CC(C)(C)O2</smiles><smiles>O=C(O)/C=C\c1cc2ccoc2cc1OC(O)C(O)C(O)C(O)CO</smiles>

102<smiles>O=C(O)/C=C/c1cc2ccoc2cc1OC(O)C(O)C(O)CO</smiles>

103<smiles>O=C(O)CCc1cc2ccoc2cc1OC1OC(CO)C(O)C(O)C1O</smiles>

104

(e)

Figure 1: Continued. 


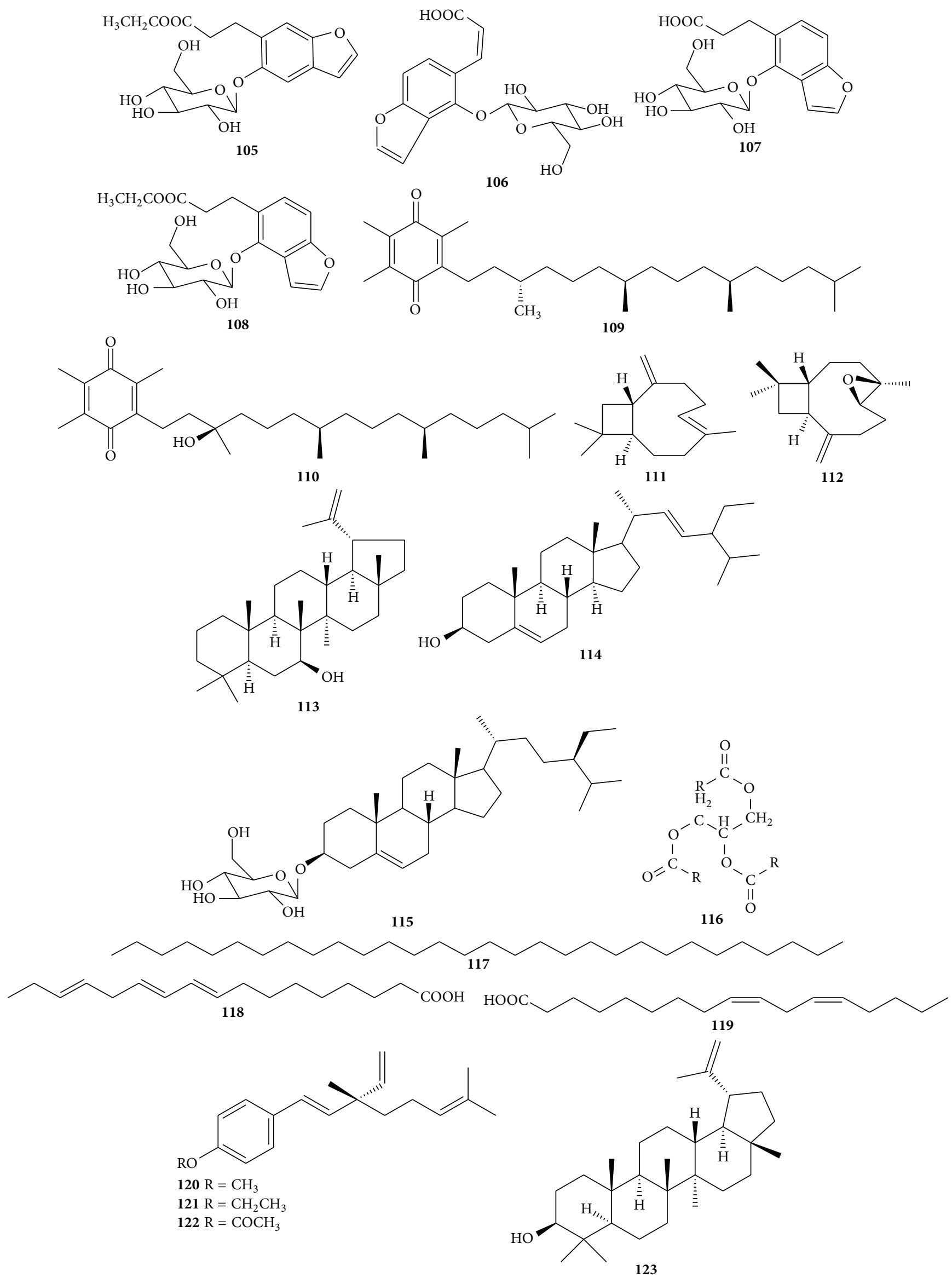

(f)

Figure 1: Continued. 
<smiles>CCCCCCCCCCCCC(=O)C[C@H]1CC/C=C\OCC(=O)CC1</smiles>

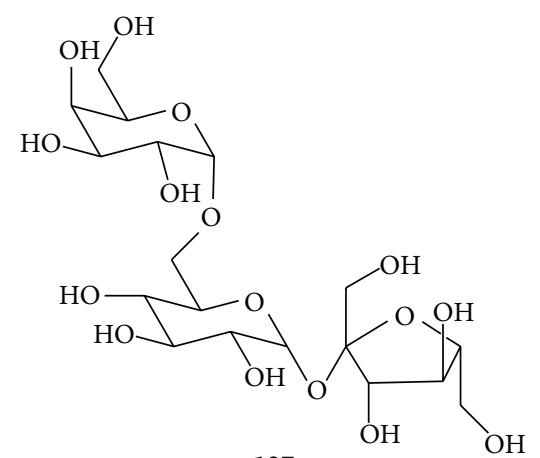
127<smiles>O=c1cc[nH]c(=O)[nH]1</smiles>

128<smiles>OCCC1OC(O)C(O)C(O)C1O</smiles>
125<smiles>COC1C(O)C(O)C2C(O)C(O)C1C2O</smiles>

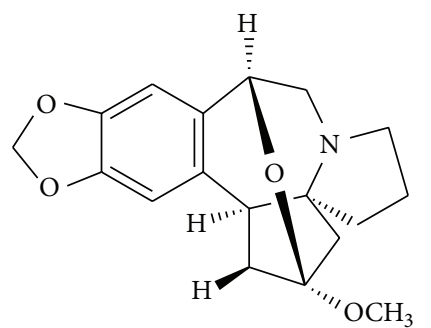

129

(g)

FIgUre 1: Chemical structures of isolated compounds from the genus Psoralea.

flavonoids, including flavones (1 5), flavonols $(6 \sim 7)$, flavanones $(8 \sim 11)$, isoflavones $(\mathbf{1 2 \sim 3 1})$, and chalcones (32 45), have been isolated and identified. According to Harborne's "The Flavonoids Advances in Research Since 1980" [6], psorachromene (46), psorachalcones A (47), 4'-O-methyl bavachalcone (48), 4,2'-dihydroxy- $2^{\prime \prime}-\left(1^{\prime \prime \prime}\right.$-methyl ethyl)$2^{\prime \prime}-3^{\prime \prime}$-dihydro- $\left(4^{\prime \prime}, 5^{\prime \prime}, 3^{\prime}, 4^{\prime}\right)$ furanochalcone $(49)$, and $7,5^{\prime \prime}$ dihydroxy-6", $6^{\prime \prime}$-dimethyl-dihydropyrano- $\left(2^{\prime \prime}, 3^{\prime \prime}, 4^{\prime}, 3^{\prime}\right)$ isoflavone (50) have the basic skeleton type of " $\mathrm{C}_{6}-\mathrm{C}_{3}-\mathrm{C}_{6}$ " of flavonoids. So in this review, this kind of compounds is classified as flavonoid.

2.2. Coumarins. Coumarin is another major type of compounds in the genus Psoralea. So far, sixteen coumarins, 51 66, have been found from the genus Psoralea. Psoralen (51), bakuchincin (54), and plicadin (61) exist both in P. corylifolia and in $P$. plicata [5, 7-14], and the others were obtained from $P$ corylifolia. In 1933, Jois got psoralen (51) from $P$. corylifolia for the first time, then Spath identified the structure and synthesized it [4]. Psoralin (65) is a special coumarin containing $\mathrm{N}$ element [10].

2.3. Phenols. To date, thirty-two phenols, 67 98, have been identified from the genus Psoralea. Five phenols, named as 3hydroxy bakuchiol (70), 12,13-dihydro-12,13-epoxy bakuchiol (74), 12-hydroxyisobakuchiol (81), and cyclobakuchiols A and $\mathrm{B}$ (84 and 85, resp.), were isolated from P. glandulosa $[49,58,59]$. Among them, 12-hydroxyisobakuchiol (81) was also found in P. corylifolia [53]. Two compounds, drupanin (72) and drupanol (75), were isolated from P. drupacea [5052]. Drupanin (72) was isolated and identified from $P$. juncea as well [51]. Five compounds $(73,78 \sim 79,97$, and 98) exist in P. plicata [5]. The other phenol derivatives were detected from P. corylifolia.
2.4. Benzofurans and Benzopyrans. Phytochemical studies have afforded ten benzofurans and benzopyrans (99 108) from the genus Psoralea. Among them, $Z$-Werneria chromenes and $E$-Werneria chromenes (100 and 101, resp.) are benzopyrans and the others are benzofurans. In 1992, one new benzofuran, named isocorylifonol (99), was found from P. corylifolia $[4,18]$. The other two compounds, psoralenoside (102) and isopsoralenoside (106), were isolated from $P$. corylifolia in 2006 [63]. Another two compounds, 107 108, were obtained from $P$. plicata [71].

2.5. Quinones. Two quinones, named $\alpha$-tocopherol quinone methyl ether and $\alpha$-tocopherol quinone (109 and $\mathbf{1 1 0}$, resp.), have been isolated from the aerial part of $P$. plicata $[1,64,65]$.

2.6. Sesquiterpenoids, Triterpenes, and Steroids. Two known sesquiterpenoids, named $\beta$-caryophyllene (111) and $\beta$-caryophyllene oxide (112), were found in P. plicata by Arafa in 1997 $[5,18]$. In 1989, Rasool and Nazli isolated a triterpene from P. plicata and named it as psoracinol (113) [1]. In addition, two steroids, named stigmasterol (114) and daucosterol (115), were isolated from $P$. corylifolia. What is more, stigmasterol (114) was identified in P. plicata as well [1].

2.7. Others. About 14 other compounds have been isolated from the genus Psoralea. Only lupeol (123) was isolated from P. plicata [1]. Drupacine (129) was detected from $P$. drupacea. Compounds 116 122 and 124 128 were isolated from $P$. corylifolia.

\section{Pharmacological Activities}

Many investigations have been conducted on the pharmacological properties of the Psoralea plants such as antimicrobial activity, antipregnancy and estrogenic activity, antitumor 
TABLE 1: Chemical constituents isolated and identified from the genus Psoralea.

\begin{tabular}{|c|c|c|c|}
\hline Number & Name & Source & Ref. \\
\hline & Flavonoids & & \\
\hline 1 & Corylifol C & P. corylifolia & {$[15]$} \\
\hline 2 & Isovitexin & P. plicata & {$[5]$} \\
\hline 3 & $4^{\prime}$-Methoxyflavone & P. corylifolia & {$[1,16]$} \\
\hline 4 & Coryfilolin=bavachin & P. corylifolia & {$[17]$} \\
\hline 5 & Bavachinin & P. corylifolia & {$[18]$} \\
\hline 6 & $\begin{array}{c}3,5,3^{\prime}, 4^{\prime} \text {-Tetrahydroxy-7-methoxyflavone- } 3^{\prime}-O-\alpha \text {-L-xylopyranosyl }(1 \rightarrow 3) \\
-O-\alpha \text {-L-arabinopyranosyl }(1 \rightarrow 4) \\
-O-\beta \text {-D-galactopyranoside }\end{array}$ & P. corylifolia & {$[17]$} \\
\hline 7 & Astragalin & P. corylifolia & {$[8]$} \\
\hline 8 & $\begin{array}{l}\text { 7,8-Dihydro-8-(4-hydroxyphenyl)-2,2- } \\
\left.\text { dimethyl-2H,6H-benzo[1,2- } b: 5,4-b^{\prime}\right] \text { dipyran-6-one }\end{array}$ & P. corylifolia & {$[15]$} \\
\hline 9 & Furan $\left(2^{\prime \prime}, 3^{\prime \prime}, 7,6\right)-4^{\prime}$-hydroxy flavanone & P. corylifolia & {$[19]$} \\
\hline 10 & Isobavachin & P. corylifolia & {$[20,21]$} \\
\hline 11 & 6-Prenylnaringenin & P. corylifolia & {$[22]$} \\
\hline 12 & Erythrinin A & P. corylifolia & {$[15]$} \\
\hline 13 & Genistein & P. corylifolia & {$[19,23]$} \\
\hline 14 & Neobavaisoflavone & P. corylifolia & $\begin{array}{l}{[1,9,15} \\
24,25]\end{array}$ \\
\hline 15 & Daidzein & P. corylifolia & {$[9]$} \\
\hline 16 & Corylinal & P. corylifolia & {$[24]$} \\
\hline 17 & Biochanin A & P. corylifolia & {$[26]$} \\
\hline 18 & Corylinal methyl ether & P. corylifolia & {$[24]$} \\
\hline 19 & 8-Prenylnaringenin & P. corylifolia & {$[15]$} \\
\hline 20 & Neobava isoflavone-7-O-methyl-ether & P. corylifolia & {$[27]$} \\
\hline 21 & Daidzin & P. corylifolia & {$[12]$} \\
\hline 22 & Bavadin & P. corylifolia & [28] \\
\hline 23 & $\begin{array}{l}\text { Corylinanl=7-O-methyl- } 3^{\prime} \text { - } \\
\text { formyl- } 4^{\prime} \text {-hydroxy isoflavone }\end{array}$ & P. corylifolia & {$[24]$} \\
\hline 24 & Corylin & P. corylifolia & $\begin{array}{l}{[1,11,15,} \\
29]\end{array}$ \\
\hline 25 & $\begin{array}{c}\text { Corylifol A } \\
=7,4^{\prime} \text {-dihydroxy-3'- }[(E)-3,7 \text {-dimethyl-2,6-octadienyl }] \text { isoflavone }\end{array}$ & P. corylifolia & {$[15]$} \\
\hline 26 & Neocorylin & P. corylifolia & {$[30]$} \\
\hline 27 & Isoneobavaisoflavone & P. corylifolia & {$[15]$} \\
\hline 28 & Psoralenol & P. corylifolia & {$[27]$} \\
\hline 29 & Psoralenol methyl ether & P. corylifolia & {$[27]$} \\
\hline 30 & Psoralenol monomethyl ether monoacetate & P. corylifolia & [27] \\
\hline 31 & Psoralenol diacetate & P. corylifolia & {$[27]$} \\
\hline 32 & Neobavachalcone $=5^{\prime}$-formyl-2',4-dihydroxy- $4^{\prime}$-methoxy chalcone & P. corylifolia & {$[31,32]$} \\
\hline 33 & Isoneobavachalcone & P. corylifolia & [33] \\
\hline 34 & Isobavachalcone=eorylifolin & P. corylifolia & {$[20,21]$} \\
\hline 35 & Bavachalcone & P. corylifolia & {$[21,34]$} \\
\hline 36 & Corylifol B & P. corylifolia & {$[35]$} \\
\hline 37 & $\begin{array}{l}4,2^{\prime} \text {-Dihydroxy- } 4^{\prime} \text {-methoxy- } 5^{\prime}-\left(3^{\prime \prime \prime}\right. \\
3^{\prime \prime \prime} \text {-dimethylallyl)chalcone }\end{array}$ & P. corylifolia & [36] \\
\hline 38 & Bakuchalcone & P. corylifolia & [37] \\
\hline 39 & Bakuchalcone & P. corylifolia & {$[37]$} \\
\hline 40 & Brosimacutin $\mathrm{G}$ & P. corylifolia & {$[15]$} \\
\hline 41 & Bavachromonol & P. corylifolia & {$[1,38]$} \\
\hline
\end{tabular}


TABle 1: Continued.

\begin{tabular}{|c|c|c|c|}
\hline Number & Name & Source & Ref. \\
\hline 42 & $\begin{array}{l}\text { 1-[2,4-Dihydroxy-3-(2-hydroxy-3-methyl-3-butenyl)phenyl]-3-(4- } \\
\text { hydroxyphenyl)-2-propen-1-one }\end{array}$ & P. corylifolia & {$[1]$} \\
\hline 43 & Psorachalcones B & P. corylifolia & {$[16]$} \\
\hline 44 & Bavachromene & P. corylifolia & {$[1,33]$} \\
\hline 45 & $\begin{array}{c}\text { Isobavachromene } \\
=4 \text {-hydroxylonchocarpin }\end{array}$ & P. corylifolia & {$[15,39]$} \\
\hline 46 & Psorachromene & P. corylifolia & {$[40]$} \\
\hline 47 & Psorachalcones A & P. corylifolia & {$[1,15]$} \\
\hline 48 & $4^{\prime}$-O-Methyl bavachalcone & P. corylifolia & [41] \\
\hline 49 & $\begin{array}{l}4,2^{\prime} \text {-Dihydroxy-2" }-\left(1^{\prime \prime \prime}-\text { methyl ethyl }\right) \\
-2^{\prime \prime}-3^{\prime \prime} \text {-dihydro- }\left(4^{\prime \prime}, 5^{\prime \prime}, 3^{\prime}, 4^{\prime}\right) \text { furanochalcone }\end{array}$ & P. corylifolia & {$[36]$} \\
\hline \multirow[t]{2}{*}{50} & $7,5^{\prime \prime}$-Dihydroxy- $6^{\prime \prime}, 6^{\prime \prime}$-dimethyl-dihydropyrano- $\left(2^{\prime \prime}, 3^{\prime \prime}, 4^{\prime}, 3^{\prime}\right)$-isoflavone & P. corylifolia & {$[24]$} \\
\hline & Coumarins & & \\
\hline 51 & Psoralen & $\begin{array}{l}\text { P. corylifolia } \\
\text { P. plicata }\end{array}$ & $\begin{array}{l}{[5,8,9,11} \\
12,14,42]\end{array}$ \\
\hline 52 & Bergapten $=5$-methoxy psoralen & P. corylifolia & {$[43]$} \\
\hline 53 & Xanthotoxin $=8$-methoxy psoralen & P. corylifolia & [43] \\
\hline 54 & Bakuchincin & $\begin{array}{l}\text { P. corylifolia } \\
\text { P. plicata }\end{array}$ & {$[5,9,10]$} \\
\hline 55 & Isopsoralen=angelicin & P. corylifolia & $\begin{array}{l}{[1,10-} \\
12,14]\end{array}$ \\
\hline 56 & Neopsoralen & P. corylifolia & {$[16]$} \\
\hline 57 & Bavacoumestan B & P. corylifolia & {$[44]$} \\
\hline 58 & Psoralidin & P. corylifolia & $\begin{array}{l}{[5,29,45-} \\
\quad 47]\end{array}$ \\
\hline 59 & Psoralidin- $2^{\prime}, 3^{\prime}$-oxide diacetate & P. corylifolia & {$[33]$} \\
\hline 60 & Isopsoralidin & P. corylifolia & {$[4]$} \\
\hline 61 & Plicadin & $\begin{array}{l}\text { P. plicata } \\
\text { P. corylifolia }\end{array}$ & {$[7,13]$} \\
\hline 62 & Corylidin & P. corylifolia & {$[1,30]$} \\
\hline 63 & Sophoracoumestan A & P. corylifolia & {$[12]$} \\
\hline 64 & Bavacoumestan A & P. corylifolia & {$[44]$} \\
\hline 65 & Psoralin & P. corylifolia & {$[10]$} \\
\hline \multirow[t]{2}{*}{66} & C-Phenylcoumarin & P. corylifolia & [33] \\
\hline & Phenols & & \\
\hline 67 & $p$-Hydroxybenzyl alcohol & P. corylifolia & {$[48]$} \\
\hline 68 & $p$-Hydroxybenzaldehyde & P. corylifolia & {$[48]$} \\
\hline 69 & p-Hydroxybenzyl acid & P. corylifolia & {$[8]$} \\
\hline 70 & 3-Hydroxy bakuchiol & P. glandulosa & [49] \\
\hline 71 & Corylifolin & P. corylifolia & {$[9,11]$} \\
\hline 72 & Drupanin & $\begin{array}{l}\text { P. drupacea } \\
\text { P. juncea }\end{array}$ & {$[50,51]$} \\
\hline 73 & Plication B & P. plicata & [32] \\
\hline 74 & 12,13-Dihydro-12,13-epoxy bakuchiol & P. glandulosa & [49] \\
\hline 75 & Drupanol & P. drupacea & {$[52]$} \\
\hline 76 & Bakuchiol & P. corylifolia & $\begin{array}{l}{[11,20,46} \\
49,53-56]\end{array}$ \\
\hline 77 & 12,13-Dihydro-12,13-dihydroxy bakuchiol & P. corylifolia & {$[54]$} \\
\hline 78 & Plicatin-A & P. plicata & {$[5,57]$} \\
\hline 79 & $\begin{array}{l}\text { Psoralea }=3 \text {-(3-methyl-2-3-epoxybutyl-)- } p \text { - } \\
\text { coumaric acid methyl ester }\end{array}$ & P. plicata & {$[5]$} \\
\hline
\end{tabular}


TABle 1: Continued.

\begin{tabular}{|c|c|c|c|}
\hline Number & Name & Source & Ref. \\
\hline 80 & 13-Hydroxyisobakuchiol & P. corylifolia & {$[53]$} \\
\hline 81 & 12-Hydroxyisobakuchiol & $\begin{array}{l}\text { P.corylifolia } \\
\text { P. glandulosa }\end{array}$ & {$[49,53]$} \\
\hline 82 & 12,13-Dihydro-12,13-epoxy bakuchiol & P. corylifolia & {$[9,54]$} \\
\hline 83 & Cyclobakuchiol C & P. corylifolia & {$[53]$} \\
\hline 84 & Cyclobakuchiols A & P. glandulosa & {$[58-60]$} \\
\hline 85 & Cyclobakuchiols B & P. glandulosa & {$[58-60]$} \\
\hline 86 & Corylifonol & P. corylifolia & {$[8]$} \\
\hline 87 & Isocorylifonol & P. corylifolia & {$[8]$} \\
\hline 88 & Psoracorylifols A & P. corylifolia & {$[34]$} \\
\hline 89 & Psoracorylifols B & P. corylifolia & {$[34]$} \\
\hline 90 & Psoracorylifols C & P. corylifolia & {$[34]$} \\
\hline 91 & Psoracorylifols D & P. corylifolia & {$[34]$} \\
\hline 92 & Psoracorylifols E & P. corylifolia & {$[34]$} \\
\hline 93 & $\alpha$-Diplicatin B & P. plicata & {$[5,61]$} \\
\hline 94 & Bisbakuchiols A & P. corylifolia & {$[54]$} \\
\hline 95 & Bisbakuchiols B & P. corylifolia & {$[54,62]$} \\
\hline 96 & Bisbakuchiols C & P. corylifolia & {$[54,62]$} \\
\hline 97 & $\alpha$-Tocopherol & P. plicata & {$[5,61]$} \\
\hline \multirow[t]{2}{*}{98} & Rososide A & P. plicata & {$[5,61]$} \\
\hline & Benzofurans and benzopyrans & & \\
\hline 99 & Isocorylifonol & P. corylifolia & {$[4,18]$} \\
\hline 100 & Z-Werneria chromenes & P. plicata & {$[5,61]$} \\
\hline 101 & E-Werneria chromenes & P. plicata & {$[5,61]$} \\
\hline 102 & Psoralenoside & P. corylifolia & {$[63]$} \\
\hline 103 & Isopsoralic acid-O-glucopyranosyl & P. plicata & {$[5,61]$} \\
\hline 104 & $1 \rightarrow 6-O-\beta$-D-Glucopyranoside isopsoralic acid & P. plicata & {$[61]$} \\
\hline 105 & $1 \rightarrow 6$-O- $\beta$-D-Glucopyranoside corylifonol & P. plicata & {$[61]$} \\
\hline 106 & Isopsoralenoside & P. corylifolia & {$[63]$} \\
\hline 107 & $\begin{array}{c}1 \rightarrow 4-O-\beta \text {-D-Glucopyranoside } \\
\text { angelic acid }\end{array}$ & P. plicata & {$[61]$} \\
\hline \multirow[t]{2}{*}{108} & $1 \rightarrow 4$-O- $\beta$-D-Glucopyranoside isocorylifonol & P. plicata & {$[61]$} \\
\hline & Quinones & & \\
\hline 109 & $\alpha$-Tocopherol quinone methyl ether & P. plicata & {$[1,7,64]$} \\
\hline \multirow[t]{2}{*}{110} & $\alpha$-Tocopherol quinone & P. plicata & {$[1,7,65]$} \\
\hline & Sesquiterpenoids, triterpenes, and steroids & & \\
\hline 111 & $\beta$-Caryophyllene & P. corylifolia & {$[18]$} \\
\hline 112 & $\beta$-Caryophyllene oxide & P. plicata & {$[5]$} \\
\hline 113 & Psoracinol & P. plicata & {$[1]$} \\
\hline 114 & Stigmasterol & $\begin{array}{l}\text { P. plicata } \\
\text { P. corylifolia }\end{array}$ & {$[4,24,66]$} \\
\hline \multirow[t]{2}{*}{115} & Daucosterol $=\beta$-sitosterol- $D$-glucoside & P. corylifolia & {$[1,67]$} \\
\hline & Others & & \\
\hline 116 & Triglyceride & P. corylifolia & {$[1,4]$} \\
\hline 117 & Triacontane & P. corylifolia & {$[1,4]$} \\
\hline 118 & Linolenic acid & P. corylifolia & {$[68]$} \\
\hline 119 & Linoleic acid & P. corylifolia & {$[68]$} \\
\hline 120 & O-Methyl bakuchiols & P. corylifolia & {$[54]$} \\
\hline 121 & O-Ethyl bakuchiols & P. corylifolia & [54] \\
\hline
\end{tabular}


TABLE 1: Continued.

\begin{tabular}{|c|c|c|c|}
\hline Number & Name & Source & Ref. \\
\hline 122 & Acetyl bakuchiol & P. corylifolia & {$[54]$} \\
\hline 123 & Lupeol & P. plicata & {$[66,69]$} \\
\hline 124 & Psoralester & P. corylifolia & {$[40]$} \\
\hline 125 & Glucose & P. corylifolia & {$[4]$} \\
\hline 126 & Pinitol & P. corylifolia & {$[16]$} \\
\hline 127 & Raffinose & P. corylifolia & {$[4]$} \\
\hline 128 & Uracil & P. corylifolia & {$[12]$} \\
\hline 129 & Drupacine $=2^{\prime}, 2^{\prime}$-dimethyl- $3^{\prime}, 4^{\prime}$-dihydropyran- $5^{\prime}, 6^{\prime}: 3,4$-trans-cinnamic acid & P. drupacea & {$[70]$} \\
\hline
\end{tabular}

activity, antioxidant activity, immunomodulatory activity, and anti-inflammatory activity.

3.1. Antimicrobial Activity. Studies have shown that the plants of genus Psoralea have significant antimicrobial activity. Yin and his colleagues tested the compounds isolated from $P$. corylifolia for antibacterial activity against two pathogenic Gram(+) bacteria Staphylococcus aureus ATCC 25923 and S. epidermidis ATCC 12228 in vitro. Among them, bavachin (4), bavachinin (5), 7,8-dihydro-8-(4-hydroxyphenyl)-2,2dimethyl-2H,6H-benzo[1,2-b:5,4- $\left.b^{\prime}\right]$ dipyran-6-one (8), erythrinin A (12), neobavaisoflavone (14), isoneobavaisoflavone (27), isobavachalcone (34), bavachalcone (35), and corylifols B (36) exhibited remarkable anti-S. aureus and anti-S. epidermidis activities at the level of MICs 0.009-0.073 mM [15]. From a literature published in 2004, bakuchincin (54), psoralidin (58), and the mixture (1:1) of angelicin (55) and psoralin (65), isolated from the seeds of $P$. corylifolia, exhibited significant antibacterial activity against Gram (+) and Gram $(-)$ bacteria as well. Particularly, angelicin (55) and psoralen (65) showed stronger activity against Gram (+) S. aureus, and psoralidin (58) inhibited Gram(-) Shigella sonnei and $S$. flexneri effectively [10]. In addition, psoracorylifols A-E (88 92), identified from the seeds of $P$. corylifolia, were reported having the inhibitory activity against Helicobacter pylori at the level of MICs of $12.5-25 \mu \mathrm{g} / \mathrm{mL}$ [34]. P. corylifolia seeds and the resinous exudate and meroterpenoids isolated from P. glandulosa had some degree of antifungal activity [72, 73]. P. glandulosa was also reported significantly inhibiting the growth of Botrytis cinerea and Phytophthora cinnamomi [74].

3.2. Antipregnancy and Estrogenic Activity. Some articles have reported that angelicin (55) and bakuchiol (76) have significant anti-implantation activity on mice $[1,18]$. And psoralidin (58), a coumestan analogue, has been considered to have a novel biological activity as an agonist for both estrogen receptor alpha $(\mathrm{ER} \alpha)$ and $\mathrm{ER} \beta$ and activate the classical ERsignaling pathway in both ER-positive human breast and endometrial cell lines as well as non-human cultured cells transiently expressing $\mathrm{ER} \alpha$ or $\mathrm{ER} \beta$ [45].

3.3. Antitumor Activity. Many researchers have investigated that the solvent extraction obtained from the plants of Psoralea has anticancer activity, especially P. corylifolia [1,
62, 75-84]. In Lee et al's research, psoralidin (58), isolated from the acetate-soluble fraction of the methanolic extract, could induce the activity of Quinone Reductase in Hepa1clc7 murine hepatoma cell line [82]. In addition, psoralidin (58) was proved to possess cytotoxity with the $\mathrm{IC}_{50}$ values of $0.3,0.4,53$, and $203 \mu \mathrm{g} / \mathrm{mL}$ against HT-29 (colon) human cancer cell line, MCF-7 (breast) human cancer cell line, SNU1 carcinoma cell line, and SNU-16 carcinoma cell line [85, 86]. Another study showed that isobavachalcone/eorylifolin (34) could induce apoptotic cell death in neuroblastoma via the mitochondrial pathway and has no cytotoxicity against normal cells, which indicated isobavachalcone/eorylifolin (34) may be applicable as an efficacious and safe drug [87]. OMethyl-bakuchiols (120) and O-ethyl-bakuchiols (121) were proved to inhibit HIF-1 ( $\mathrm{IC}_{50}$ values: 8.7 and $26.3 \mu \mathrm{M}$, resp.) and $\mathrm{NF}-\kappa \mathrm{B}$ ( $\mathrm{IC}_{50}$ values: 5.7 and $12.2 \mu \mathrm{M}$, resp.) activation without significantly decreasing the viability of the human gastric cancer cell and human cervical adenocarcinoma cell, respectively [54]. The ethanolic extract of $P$. corylifolia was found to be cytotoxic against L929-cells in cell culture. Bakuchiol (76) was responsible for the activity [88-90].

3.4. Antioxidant Activity. There is considerable interest in more potent antioxidant compounds to treat diseases involving oxidative stress [18]. When examined for the antioxidant activity using the 2,2V-azinobis[3-ethylbenzothiazoline-6sulfonate] (ABTS) assay, $P$. corylifolia seed's solvent extract showed higher antioxidant activity [91]. In Jiangning et al's research, the powder and extracts of $P$. corylifolia were investigated in lard at $100^{\circ} \mathrm{C}$ by using Oxidative Stability Instrument (OSI) and were proved to have strong antioxidant activity. When the compounds isolated from $P$. corylifolia are tested individually and compared with butylated hydroxytoluene (BHT) and $\alpha$-tocopherol by the OSI at $100^{\circ} \mathrm{C}$, corylin (24), psoralidin (58), and bakuchiol (76) showed strong antioxidant activity, and especially psoralidin (58) (stronger antioxidant property than BHT). The specific antioxidant effect of the compounds decreases in the following order: psoralidin (58) $>$ BHT $>\alpha$-tocopherol $>$ bakuchiol (76) $>$ corylifolin (71) $>$ corylin $(24)>$ isopsoralen/angelicin (55) $\sim$ psoralen (51) [11]. Isobavachin (10) and isobavachalcone/eorylifolin (34) were proved to have broad antioxidative activities in rat liver microsomes and mitochondria [91]. In addition, the relationship between isoflavones and their antioxidant 
activities in $P$. corylifolia was studied and the research determined the antioxidant activity of extracts using 1,1-diphenyl2-picrylhydrazyl (DPPH) radical scavenging and phosphomolybdenum assays; as a result, the antioxidant activities were correlated with the content of total phenolics in the extracts [8]. In another study, some antioxidant components were isolated from $P$. corylifolia by a combinative method using high-speed countercurrent chromatography (HSCCC) and thin layer chromatography (TLC) as an antioxidant autographic assay [92].

3.5. Immunomodulatory Activity. Polysaccharide was reported to enhance the immunity of mice [8]. Wang et al.s experiments have shown that $P$. corylifolia could effectively increase the proliferation rate of diploid fibroblasts and increase the ability of nonspecific immunity [93]. The flavonoids isolated from $P$. corylifolia have also been shown to have immunological function [94]. In another study, the seeds extracts of $P$. corylifolia obtained in alcohol have been found to stimulate the immune system in mice by increasing cell mediated and humoral immune responses [77].

3.6. Anti-Inflammatory Activity. The petroleum ether extract, dichloromethane extract, and methanol extract of the aerial part of $P$. glandulosa had significant anti-inflammatory activity [95]. Another study has reported that bakuchiol (76) from Psoralea corylifolia could inhibit the expression of inducible nitric oxide synthase (iNOS) gene via the inactivation of nuclear transcription factor- $\kappa \mathrm{B}$ in RAW 264.7 macrophages [96].

3.7. Antimutagenic Activity. Several flavonoids isolated from P. corylifolia have the antimutagenic activity $[97,98]$.

3.8. Antiviral Activity. The volatiles isolated from P. drupacea's leaves and stem barks have antiviral activity [1].

3.9. Hepatoprotective Activity. P. corylifolia has significant hepatoprotective activity $[99,100]$. Bakuchiol (76), bakuchincin (54), and psoralen (51) have been proved to be hepatoprotective with $\mathrm{EC}_{50}$ values of $1.0,47.0$, and $50.0 \mu \mathrm{g} / \mathrm{mL}$, respectively, on tacrine-induced cytotoxicity in human liverderived Hep G2 cells using silymarin as a positive control with $\mathrm{EC}_{50}$ value of $5.0 \mu \mathrm{g} / \mathrm{mL}[101]$.

3.10. Photosensitization. Ethanol extract of $P$. corylifolia has an effect on tyrosinase and increases the volume and speed of melanin by improving the activity of tyrosinase [102, 103]. Isopsoralen/angelicin (55) has been known as photosensitivity [4]. Psoralen (51) is a photosensitive compound, and its photosensitivity is much better than isopsoralen (55). It plays a key role in treating vitiligo. In addition, psoralen (51) has good effect on treating psoriasis and alopecia areata $[4,104]$.

3.11. Antiasthma Activity. Experiments have shown that coumarins isolated from $P$. corylifolia had antiasthma activity $[105,106]$. In another study, a Chinese herbal decoction, which contains 6 herbs, along with $15 \mathrm{~g}$ seeds of $P$. corylifolia, could prompt treatment for asthma in the convalescent stage to prevent emphysema [107].
3.12. Antifilarial Activity. Qamaruddin et al. reported that the aqueous and alcohol extracts of the leaves and seeds of $P$. corylifolia possessed significant antifilarial activity against Setaria cervi [108]. The extracts caused the inhibition of spontaneous movements of the whole worm and the nerve muscle preparation of S. cervi [108].

3.13. Antiplatelet Activity. The methanolic extract of seeds of P. corylifolia was identified to inhibit the aggregation of rabbit platelets induced by arachidonic acid, collagen, and platelet activating factor [109].

3.14. Osteoblastic Activity. P. corylifolia has significant inhibition effect on osteoclast $[110,111]$. Corylin (24) and bavachin/coryfilolin (4) were reported to promote the proliferation of osteoblasts and inhibit bone resorption [112]. Solvent extract, especially bakuchiol (76), had preventive effect on osteoporosis which is caused by estrogen deficiency $[113,114]$.

3.15. Hemostatic Activity. There have been some reports on whether isopsoralen/angelicin (55) possessed significant hemostatic activity [4].

3.16. Antipyretic Activity. The petroleum ether extract, dichloromethane extract, and methanol extract of the aerial part of $P$. glandulosa have antipyretic activity [95].

3.17. Antidepressant Activity. The coumarins, isolated from $P$. corylifolia, could exert antidepressant effect by regulating monoamine oxidase activity, hypothalamic-pituitary-adrenal axis function, and oxidative stress [115-117]. Psoralen (51), a major furocoumarin isolated from $P$. corylifolia, could significantly reduce immobility and increase swimming without altering climbing in the mouse forced swimming test (FST). Psoralen remarkably reversed FST-induced alterations in serotonin (5-HT) and 5-hydroxyindoleacetic acid (5HIAA) levels in frontal cortex and hippocampus in mice. Furthermore, psoralen attenuated FST-induced elevations in serum corticotropin-releasing factor (CRF) and corticosterone concentrations to normalize the HPA axis activity [118].

3.18. Others. Psoralen (51) can enhance the synthesis of prostaglandin and give priority to increasing $\mathrm{PGF}_{2 \alpha}$ [119]. It can also treat Alzheimer's disease [120]. In addition, P. corylifolia have antiaging activity [121], pesticidal activity [122], antidiabetic activity [123], antihypercholesterolemic activity [124], antiulcer activity, and so on [110].

\section{Conclusion}

Although the genus Psoralea contains more than 130 species in the world, only several plants were chemically and pharmacologically reported in the past literatures. Up to 2015, 129 compounds have been isolated from this genus. Among them, flavonoids (50 compounds) are the characteristic constituents, and coumarins, phenols, benzofurans and benzofurans glycosides, quinines, meroterpene phenols, sesquiterpenoids, and triterpenes are also found in the genus. 
The pharmacological activities, for example, antimicrobial, antitumor, antioxidant, immunomodulatory, anti-inflammatory, hepatoprotective, photosensitization, and antiasthma activities, have been often reported in the past few decades. In this review, we compiled the pharmacological activities of the extracts and the compounds from the plants of genus Psoralea. We believe there will be more researches on this genus in the future, and the bioactive constituents from this genus await further investigation.

\section{Competing Interests}

The authors declare no conflict of interests.

\section{Authors' Contributions}

Cong-Cong Li and Teng-Long Wang contributed equally to this work.

\section{Acknowledgments}

This work was supported financially by the National Natural Science Foundation (nos. 81403059 and 81403060), Tianjin Science and Technology Commissioner Project (no. 16JCTPJC49000), and the National Science and Technology Major Projects for "Major New Drugs Innovation and Development” (2014ZX09304307-001-005).

\section{References}

[1] Y. J. Bai, Y. J. Han, J. D. Fan, and Y. Y. Zhao, "Studies on the chemical constituents and pharmacological activities of genus Psoralea," in National Medicine Plants and Traditional, pp. 4249, Chinese Medicine Academic Forum, Dalian, China, 2010.

[2] L. M. Perry and J. Metzger, "Medicinal plants of east and southeast Asia: attributed properties and uses," Brittonia, vol. 33, no. 2, pp. 447-493, 1981.

[3] Chinese Pharmacopoeia Commission, Pharmacopoeia of People's Republic of China, vol. 1, China Medical Science Press, Beijing, China, 2005.

[4] L. Ji and Z. Xu, "Review of constituents in fruits of Psoralea corylifolia L.," China Journal of Chinese Materia Medica, vol. 20, no. 2, pp. 120-128, 1995.

[5] A. I. Hamed, I. Springuel, N. A. El-Emary, H. Mitome, and Y. Yamada, "A phenolic cinnamate dimer from Psoralea plicata," Phytochemistry, vol. 45, no. 6, pp. 1257-1261, 1997.

[6] C. F. Timberlake, "The flavonoids-advances in research since 1980: edited by J. B. Harborne. Chapman \& Hall, London and New York, xiii+621 pp. £95," Phytochemistry, vol. 28, no. 3, p. 966, 1989.

[7] N. Rasool, A. Q. Khan, V. U. Ahmad, and A. Malik, "A benzoquinone and a coumestan from Psoralea plicata," Phytochemistry, vol. 30, no. 8, pp. 2800-2803, 1991.

[8] Y.-L. Lin and Y.-H. Kuo, "Two new benzofuran derivatives, corylifonol and isocorylifonol from the seeds of Psoralea corylifolia," Heterocycles, vol. 34, no. 8, pp. 1555-1564, 1992.

[9] N. J. Sun, S. H. Woo, J. M. Cassady, and R. M. Snapka, "DNA polymerase and topoisomerase II inhibitors from Psoralea corylifolia," Journal of Natural Products, vol. 61, no. 3, pp. 362366, 1998.
[10] N. A. Khatune, M. E. Islam, M. E. Haque, P. Khondkar, and M. M. Rahman, "Antibacterial compounds from the seeds of Psoralea corylifolia," Fitoterapia, vol. 75, no. 2, pp. 228-230, 2004.

[11] G. Jiangning, W. Xinchu, W. Hou, L. Qinghua, and B. Kaishun, "Antioxidants from a Chinese medicinal herb-Psoralea corylifolia L.," Food Chemistry, vol. 91, no. 2, pp. 287-292, 2005.

[12] B. Ruan, L.-Y. Kong, Y. Takaya, and M. Niwa, "Studies on the chemical constituents of Psoralea corylifolia L.," Journal of Asian Natural Products Research, vol. 9, no. 1, pp. 41-44, 2007.

[13] S.-M. Wei, Z.-Z. Yan, and J. Zhou, "Psoralea corylifolia protects against testicular torsion/detorsion-induced ischemia/reperfusion injury," Journal of Ethnopharmacology, vol. 137, no. 1, pp. 568-574, 2011.

[14] R. Liu, A. Li, A. Sun, and L. Kong, "Preparative isolation and purification of psoralen and isopsoralen from Psoralea corylifolia by high-speed counter-current chromatography," Journal of Chromatography A, vol. 1057, no. 1-2, pp. 225-228, 2004.

[15] S. Yin, C. X. Fan, Y. Wang, L. Dong, and J. M. Yue, "Antibacterial prenylflavone derivatives from Psoralea corylifolia, and their structure-activity relationship study," Bioorganic \& Medicinal Chemistry, vol. 12, no. 16, pp. 4387-4392, 2004.

[16] L. L. Yu, Studies on Chemical Constituents of Psoralea corylifolia L. Aristolochia Contorta and Valerinan jatamaisi Jones, Yunnan Normal Uiversity, 2004.

[17] R. N. Yadava and V. Verma, "A new biologically active flavonol glycoside from Psoralea corylifolia (Linn.)," Journal of Asian Natural Products Research, vol. 7, no. 4, pp. 671-675, 2005.

[18] B. Chopra, A. K. Dhingra, and K. L. Dhar, "Psoralea corylifolia L. (Buguchi)-folklore to modern evidence: review," Fitoterapia, vol. 90, pp. 44-56, 2013.

[19] H. Liu, Y.-J. Bai, Y.-Y. Chen, and Y.-Y. Zhao, "Studies on chemical constituents from seed of Psoralea corylifolia," China Journal of Chinese Materia Medicine, vol. 33, no. 12, pp. 1410-1412, 2008.

[20] H. Haraguchi, J. Inoue, Y. Tamura, and K. Mizutani, "Antioxidative components of Psoralea corylifolia (Leguminosae)," Phytotherapy Research, vol. 16, no. 6, pp. 539-544, 2002.

[21] V. K. Bhalla, U. R. Nayak, and S. Dev, "Some new flavonoids from Psoralea corylifolia," Tetrahedron Letters, vol. 24, no. 20, pp. 2401-2406, 1968.

[22] Y. J. Kim, H. Lee, E. Park, and S. H. Shim, "Inhibition of human $20 S$ proteasome by compounds from seeds of Psoralea corylifolia," Bulletin of the Korean Chemical Society, vol. 30, no. 8, pp. 1867-1869, 2009.

[23] W. L. Ji, M. J. Qin, and Z. T. Wang, "Studies on the chemical constituents of Belamcanda chinesensis (I)," Journal of China Pharmaceutical University, vol. 32, no. 3, pp. 197-199, 2001.

[24] G. K. Gupta, K. L. Dhar, and C. K. Atal, "Corylinal: a new isoflavone from seeds of Psoralea corylifolia," Phytochemistry, vol. 17, no. 1, p. 164, 1978.

[25] B. S. Bajwa, P. L. Khanna, and T. R. Seshadri, "New chromenochalcone bavachromene from the seeds of Psoralea corylifolia," Current Science, vol. 41, no. 22, pp. 814-815, 1972.

[26] Y.-T. Hsu, C.-J. Wu, J.-M. Chen, Y.-C. Yang, and S.-Y. Wang, "The presence of three isoflavonoid compounds in Psoralea corylifolia," Journal of Chromatographic Science, vol. 39, no. 10, pp. 441-444, 2001.

[27] J. L. Suri, G. K. Gupta, K. L. Dhar, and C. K. Atal, "Psoralenol: a new isoflavone from the seeds of Psoralea corylifolia," Phytochemistry, vol. 17, no. 11, p. 2046, 1978.

[28] T.-T. Yang and M.-J. Qin, "Isolation and structure identification of a new isoflavone from Psoralea corylifolia," Yao Xue Xue Bao, vol. 41, no. 1, pp. 76-79, 2006. 
[29] L. H. Zhao, C. Y. Huang, Z. Shan, B. R. Xiang, and L. H. Mei, "Fingerprint analysis of Psoralea corylifolia L. by HPLC and LCMS," Journal of Chromatography B: Analytical Technologies in the Biomedical \& Life Sciences, vol. 821, no. 1, pp. 67-74, 2005.

[30] G. K. Gupta, K. L. Dhar, and C. K. Atal, "Isolation and constitution of corylidin: a new coumestrol from the fruits of Psoralea corylifolia," Phytochemistry, vol. 16, no. 3, pp. 403-404, 1977.

[31] S. R. Gupta, T. R. Seshadri, and G. R. Sood, "New formylated chalkone, $5^{\prime}$-formyl-2',4-dihydroxy-4' -methoxychalkone, from the seeds of Psoralea corylifolia," Indian Journal of Chemistry, vol. 13, no. 6, p. 632, 1975.

[32] S. R. Gupta, T. R. Seshadri, and G. R. Sood, “The structure and synthesis of neobavachalcone, a new component of Psoralea corylifolia," Phytochemistry, vol. 16, no. 12, pp. 1995-1997, 1997.

[33] B. K. Gupta, G. K. Gupta, K. L. Dhar, and C. K. Atal, "A Cformylated chalcone from Psoralea corylifolia," Phytochemistry, vol. 19, no. 9, pp. 2034-2035, 1980.

[34] S. Yin, C.-Q. Fan, L. Dong, and J.-M. Yue, "Psoracorylifols A-E, five novel compounds with activity against Helicobacter pylori from seeds of Psoralea corylifolia," Tetrahedron, vol. 62, no. 11, pp. 2569-2575, 2006.

[35] L. L. Yu, Y. G. Chen, Y. P. Lu, and S. H. Gui, "Chalcones from the seeds of Psoralea corylifolia," ChemInform, vol. 36, no. 48, pp. 1173-1177, 2005.

[36] D. Agarwal, S. P. Garg, and P. Sah, "Isolation of chalkones from the seeds of Psoralea corylifolia Linn," Indian Journal of Chemistry-Section B Organic and Medicinal Chemistry, vol. 45, no. 11, pp. 2574-2579, 2006.

[37] G. K. Gupta, J. L. Suri, B. K. Gupta, and K. L. Dhar, "Bakuchalcone, a dihydrofuranochalcone from the seeds of Psoralea corylifolia," Phytochemistry, vol. 21, no. 8, pp. 2149-2151, 1982.

[38] J. L. Suri, G. K. Gupta, K. L. Dhar, and C. K. Atal, "Bavachromanol: a new chalcone from the seeds of Psoralea corylifolia," Phytochemistry, vol. 19, no. 2, pp. 336-337, 1980.

[39] M. H. Lee, J. Y. Kim, and J.-H. Ryu, "Prenylflavones from Psoralea corylifolia inhibit nitric oxide synthase expression through the inhibition of I- $\kappa \mathrm{B}-\alpha$ degradation in activated microglial cells," Biological \& Pharmaceutical Bulletin, vol. 28, no. 12, pp. 2253-2257, 2005.

[40] A. Tewari and R. S. Bhakuni, "New constituents from Psoralea corylifolia," Indian Journal of Chemistry-Section B Organic and Medicinal Chemistry, vol. 49, no. 2, pp. 256-259, 2010.

[41] L. N. Shan, S. M. Yang, G. Zhang et al., "Comparison of the inhibitory potential of bavachalcone and corylin against UDP-glucuronosyltransferases," Evidence-Based Complementary and Alternative Medicine, vol. 2014, Article ID 958937, 6 pages, 2014.

[42] Y.-H. Kuo and Y.-L. Lin, "Two new benzofuran derivatives, corylifonol and isocorylifonol from the seeds of Psoralea corylifolia," Heterocycles, vol. 34, no. 8, pp. 1555-1564, 1992.

[43] M. A. Pathak, T. B. Fitzpatrick, and T. B. Fitzpatrick, “The presently known distribution of furocoumarins (psoralens) in plants," Journal of Investigative Dermatology, vol. 39, no. 3, pp. 225-239, 1963.

[44] S. Gupta, B. N. Jha, G. K. Gupta, B. K. Gupta, and K. L. Dhar, "Coumestans from seeds of Psoralea corylifolia," Phytochemistry, vol. 29, no. 7, pp. 2371-2373, 1990.

[45] X. Y. Liu, J.-W. Nam, Y. S. Song et al., "Psoralidin, a coumestan analogue, as a novel potent estrogen receptor signaling molecule isolated from Psoralea corylifolia," Bioorganic and Medicinal Chemistry Letters, vol. 24, no. 5, pp. 1403-1406, 2014.
[46] S. M. Newton, C. Lau, S. S. Gurcha, G. S. Besra, and C. W. Wright, "The evaluation of forty-three plant species for in vitro antimycobacterial activities; isolation of active constituents from Psoralea corylifolia and Sanguinaria canadensis," Journal of Ethnopharmacology, vol. 79, no. 1, pp. 57-67, 2002.

[47] H. N. Khastgir, P. C. Duttagupta, and P. Sengupta, "The structure of psoralidin," Tetrahedron, vol. 14, no. 3-4, pp. 275283, 1961.

[48] G. P. Peng, P. H. Wu, H. Y. Li, and Y. T. Yuan, "Chemical studies on Psoralea corylifolia," Journal of Chinese Medicine Materia, vol. 25, no. 11, pp. 563-565, 1996.

[49] C. Labbé, F. Faini, J. Coll, and J. D. Connolly, "Bakuchiol derivatives from the leaves of Psoralea glandulosa," Phytochemistry, vol. 42, no. 5, pp. 1299-1303, 1996.

[50] A. Schmitt, H. Telikepalli, and L. A. Mitscher, "Plicatin B, the antimicrobial principle of Psoralea juncea," Phytochemistry, vol. 30, no. 11, pp. 3569-3570, 1991.

[51] L. A. Golovina and G. K. Nikonov, "The structures of drupacin and drupanin-new components of Psoralea drupaceae," Chemistry of Natural Compounds, vol. 9, no. 6, pp. 672-675, 1975.

[52] L. A. Golovina and G. K. Nikonov, "The structure of drupanola new phenol from Psoralea drupaceae," Chemistry of Natural Compounds, vol. 9, no. 1, pp. 7-9, 1975.

[53] S. Yin, C.-Q. Fan, and J.-M. Yue, "Cyclobakuchiol C, a new bakuchiol derivative from Psoralea coryllfolia," Journal of Asian Natural Products Research, vol. 9, no. 1, pp. 29-33, 2007.

[54] C.-Z. Wu, S. S. Hong, X. F. Cai et al., "Hypoxia-inducible factor1 and nuclear factor $-\kappa \mathrm{B}$ inhibitory meroterpene analogues of bakuchiol, a constituent of the seeds of Psoralea corylifolia," Bioorganic \& Medicinal Chemistry Letters, vol. 18, no. 8, pp. 2619-2623, 2008.

[55] G. Mehta, U. R. Nayak, and S. Dev, "Meroterpenoids-I: Psoralea corylifolia Linn. - 1. bakuchiol, a novel monoterpene phenol," Tetrahedron, vol. 29, no. 8, pp. 1119-1125, 1973.

[56] R. K. Chaudhuri and K. Bojanowski, "Bakuchiol: a retinollike functional compound revealed by gene expression profiling and clinically proven to have anti-aging effects," International Journal of Cosmetic Science, vol. 36, no. 3, pp. 221-230, 2014.

[57] N. Rasool, A. Q. Khan, and A. Malik, "Plicatin A and B, two phenolic cinnamates from Psoralea plicata," Phytochemistry, vol. 29, no. 12, pp. 3979-3981, 1990.

[58] A. N. Shinde, N. Malpathak, and D. P. Fulzele, "Determination of isoflavone content and antioxidant activity in Psoralea corylifolia L. callus cultures," Food Chemistry, vol. 118, no. 1, pp. 128$132,2010$.

[59] C. N. Backhouse, C. L. Delporte, R. E. Negrete et al., "Cyclobakuchiols A and B from Psoralea Glandulosa," Phytochemistry, vol. 40, no. 1, pp. 325-327, 1995.

[60] C. N. Backhouse, C. L. Delporte, R. E. Negrete et al., "Cyclobakuchiols A and B from Psoralea glandulosa," Phytochemistry, vol. 40, no. 1, pp. 325-327, 1995.

[61] A. I. Hamed, I. V. Springuel, and N. A. El-Emary, "Benzofuran glycosides from the seeds of Psoralea plicata Del," Studies in Plant Science, vol. 6, pp. 323-329, 1999.

[62] C.-Z. Wu, X. F. Cai, N. T. Dat et al., "Bisbakuchiols A and B, novel dimeric meroterpenoids from Psoralea corylifolia," Tetrahedron Letters, vol. 48, no. 50, pp. 8861-8864, 2007.

[63] C.-F. Qiao, Q.-B. Han, S.-F. Mo et al., "Psoralenoside and isopsoralenoside, two new benzofuran glycosides from Psoralea corylifolia," Chemical \& Pharmaceutical Bulletin, vol. 54, no. 5, pp. 714-716, 2006. 
[64] F. J. Rupérez, D. Martín, E. Herrera, and C. Barbas, “Chromatographic analysis of $\alpha$-tocopherol and related compounds in various matrices," Journal of Chromatography A, vol. 935, no. 12, pp. 45-69, 2001.

[65] D.-S. Yang, Z.-L. Li, W.-B. Peng et al., "Three new prenylated flavonoids from Macaranga denticulata and their anticancer effects," Fitoterapia, vol. 103, pp. 165-170, 2015.

[66] D. Rathee, S. Rathee, P. Rathee, A. Deep, S. Anandjiwala, and D. Rathee, "HPTLC densitometric quantification of stigmasterol and lupeol from Ficus religiosa," Arabian Journal of Chemistry, vol. 8, no. 3, pp. 366-371, 2015.

[67] J. Huang, L. H. Zhao, Q. G. Zou, and B. R. Xiang, "New developments of chemical and pharmcological study on Psoralea," Progress in Pharmaceutical Sciences, vol. 2000, no. 4, pp. 212214, 2000 .

[68] H. Matsuda, S. Sugimoto, T. Morikawa et al., "Bioactive constituents from Chinese natural medicines. XX. ${ }^{1}$ ) inhibitors of antigen-induced degranulation in RBL-2H3 Ccells from the seeds of Psoralea corylifolia," Chemical \& Pharmaceutical Bulletin, vol. 55, no. 1, pp. 106-110, 2007.

[69] J. A. Sánchez-Burgos, M. V. Ramírez-Mares, J. A. GallegosInfante et al., "Isolation of lupeol from white oak leaves and its anti-inflammatory activity," Industrial Crops and Products, vol. 77, no. 23, pp. 827-832, 2015.

[70] I. Takano, I. Yasuda, M. Nishijima, Y. Hitotsuyanagi, K. Takeya, and H. Itokawa, "Drupangtonine, a novel antileukemic alkaloid from Cephalotaxus harringtonia var. drupacea," Bioorganic \& Medicinal Chemistry Letters, vol. 6, no. 14, pp. 1689-1690, 1996.

[71] W. M. Yang, M. S. Chang, and S. K. Park, "Effects of Psoralea corylifolia on the cAMP-responsive element modulator (CREM) expression and spermatogenesis in rats," Journal of Ethnopharmacology, vol. 117, no. 3, pp. 503-506, 2008.

[72] A. Madrid, L. Espinoza, C. González et al., "Antifungal study of the resinous exudate and of meroterpenoids isolated from Psoralea glandulosa (Fabaceae)," Journal of Ethnopharmacology, vol. 144, no. 3, pp. 809-811, 2012.

[73] X. Y. Yang, J. Li, X. W. Wang et al., "Psc-AFP, an antifungal protein with trypsin inhibitor activity from Psoralea corylifolia seeds," Peptides, vol. 27, no. 7, pp. 1726-1731, 2006.

[74] A. M. Villegas, K. D. Peralta, C. G. Tapia, K. C. Marín, and L. E. Catalán, "Antiphytopathogenic activity of Psoralea glandulosa (Fabaceae) against Botrytis cinerea and Phytophthora cinnamomi," Natural Product Research, vol. 29, no. 6, pp. 586-588, 2015.

[75] Y. Wang, C. Hong, C. Zhou, D. Xu, and H.-B. Qu, "Screening antitumor compounds psoralen and isopsoralen from Psoralea corylifolia L. seeds," Evidence-Based Complementary and Alternative Medicine, vol. 2011, Article ID 363052, 7 pages, 2011.

[76] K. Bapat, G. J. Chintalwar, U. Pandey et al., "Preparation and in vitro evaluation of radioiodinated bakuchiol as an anti tumor agent," Applied Radiation \& Isotopes, vol. 62, no. 3, pp. 389-393, 2005.

[77] P. G. Latha, D. A. Evans, K. R. Panikkar, and K. K. Jayavardhanan, "Immunomodulatory and antitumour properties of Psoralea corylifolia seeds," Fitoterapia, vol. 71, no. 3, pp. 223-231, 2000.

[78] J. N. Guo, H. Wu, X. C. Weng, J. H. Yan, and K. S. Bi, "Studies on extraction and isolation of active constituents from Psoralen corylifolia L. and the antitumor effect of the constituents in vitro," Journal of Chinese Medicinal Materials, vol. 26, no. 3, pp. 185-187, 2003.
[79] L. C. Whelan and M. F. Ryan, "Ethanolic extracts of Euphorbia and other ethnobotanical species as inhibitors of human tumour cell growth," Phytomedicine, vol. 10, no. 1, pp. 53-58, 2003.

[80] S. Y. Tang, J. Gruber, K. P. Wong, and B. Halliwell, "Psoralea corylifolia L. inhibits mitochondrial complex I and proteasome activities in SH-SY5Y cells," Annals of the New York Academy of Sciences, vol. 1100, no. 1, pp. 486-496, 2007.

[81] S. Y. Ryu, S. U. Choi, C. O. Lee, and O. P. Zee, "Antitumor activity of Psoralea corylifolia," Archives of Pharmacal Research, vol. 15, no. 4, pp. 356-359, 1992.

[82] S.-J. Lee, K.-W. Nam, and W. Mar, "Induction of quinone reductase activity by psoralidin isolated from Psoralea corylifolia in mouse hepa 1clc7 cells," Archives of Pharmacal Research, vol. 32, no. 7, pp. 1061-1065, 2009.

[83] S.-J. Lee, K.-W. Nam, and W. Mar, "Effects of psoralen from Psoralea corylifolia on quinone reductase, ornithine decarboxylase, and JB6 cells transformation promotion," Archives of Pharmacal Research, vol. 34, no. 1, pp. 31-36, 2011.

[84] P. G. Latha and K. R. Panikkar, "Inhibition of chemical carcinogenesis by Psoralea corylifolia seeds," Journal of Ethnopharmacology, vol. 68, no. 1-3, pp. 295-298, 1999.

[85] Y.-M. Yang, J.-W. Hyun, M.-S. Sung et al., "The cytotoxicity of psoralidin from Psoralea corylifolia," Planta Medica, vol. 62, no. 4, pp. 353-354, 1996.

[86] W. Mar, K. H. Je, and E. K. Seo, "Cytotoxic constituents of Psoralea corylifolia," Archives of Pharmacal Research, vol. 24, no. 3, pp. 211-213, 2001.

[87] R. Nishimura, K. Tabata, M. Arakawa et al., "Isobavachalcone, a chalcone constituent of Angelica keiskei, induces apoptosis in neuroblastoma," Biological \& Pharmaceutical Bulletin, vol. 30, no. 10, pp. 1878-1883, 2007.

[88] J. Iwamura, T. Dohi, H. Tanaka, T. Odani, and M. Kubo, "Cytotoxicity of corylifoliae fructus. II. Cytotoxicity of bakuchiol and the analogues," Journal of the Pharmaceutical Society of Japan, vol. 109, no. 12, pp. 962-965, 1989.

[89] M. Kubo, T. Dohi, T. Odani, H. Tanaka, and J. Iwamura, "Cytotoxicity of corylifoliae fructus. I. Isolation of the effective compound and the cytotoxicity," Yakugaku Zasshi Journal of the Pharmaceutical Society of Japan, vol. 109, no. 12, pp. 926-931, 1989.

[90] V. D. Rangari and S. R. Agrawal, "Chemistry and pharmacology of Psoralea corylifolia," Indian Drugs, vol. 29, no. 15, pp. 662-670, 1992.

[91] S. Y. Tang, M. Whiteman, Z. F. Peng, A. Jenner, E. L. Yong, and B. Halliwell, "Characterization of antioxidant and antiglycation properties and isolation of active ingredients from traditional Chinese medicines," Free Radical Biology \& Medicine, vol. 36, no. 12, pp. 1575-1587, 2004.

[92] G. D. Xiao, G. W. Li, L. Chen et al., "Isolation of antioxidants from Psoralea corylifolia fruits using high-speed countercurrent chromatography guided by thin layer chromatographyantioxidant autographic assay," Journal of Chromatography A, vol. 1217, no. 34, pp. 5470-5476, 2010.

[93] S. L. Wang, S. D. Wu, S. L. Li, H. Xiao, C. T. Dong, and L. Cui, "Experimental study on the effect of fructus lyc II and 7 other Chinese drugs on cells cultured in vitro and macrophages of the abdominal cavity in mice," Journal of Norman Bethune University of Medical Sciences, vol. 4, 1990.

[94] Y. H. Qin, Y. Cui, Y. X. Ren et al., "Immunological regulation and treatment of brucea javanica and fructus Psoraleae on 
rats with Pneumocystis carinii pneumonia," Chinese Journal of Parasitology and Parasitic Diseases, vol. 24, no. 1, pp. 59-62, 2006.

[95] C. N. Backhouse, C. L. Delporte, R. E. Negrete et al., "Active constituents isolated from Psoralea glandulosa L. with antiinflammatory and antipyretic activities," Journal of Ethnopharmacology, vol. 78, no. 1, pp. 27-31, 2001.

[96] H.-O. Pae, H. Cho, G.-S. Oh et al., "Bakuchiol from Psoralea corylifolia inhibits the expression of inducible nitric oxide synthase gene via the inactivation of nuclear transcription factor$\kappa$ B in RAW 264.7 macrophages," International Immunopharmacology, vol. 1, no. 9-10, pp. 1849-1855, 2001.

[97] M. E. Wall, M. C. Wani, G. Manikumar et al., "Plant antimutagenic agents, 2. Flavonoids," Journal of Natural Products, vol. 51, no. 6, pp. 1084-1091, 1988.

[98] J. S. Ye, L. Y. Shen, Z. G. Li, and S. R. Cai, "Antioxidative protection of water extract of Psoralea fruit and bakuchiol on primary rat hepatocytes," Nutrition Science Journal, vol. 22, no. 5, pp. 303-324, 1997.

[99] S. M. Ye, W. P. Ou, and X. Hong, "Pharmacokinetics of fructus Psoraleae in bile elimination," Traditinal Chinese Drug Research \& Clinical Pharmacology, vol. 10, no. 3, pp. 162-164, 1999.

[100] Z. Dai, C. Zhao, K. Zhou, C. Q. Qu, and L. M. Hu, "Effect of fructus Psoraleae on rats' bile secretion and contractile activity $\mathrm{f}$ guinea-pigs' gallbladder smooth muscle," Strait Pharmaceutical Journal, vol. 22, no. 4, pp. 37-38, 2009.

[101] H. Cho, J.-Y. Jun, E.-K. Song et al., "Bakuchiol: a hepatoprotective compound of Psoralea corylifolia on tacrine-induced cytotoxicity in hep G2 cells," Planta Medica, vol. 67, no. 8, pp. 750751, 2001.

[102] J. G. Xu and J. Shang, "The activition of Psoralea corylifolia on tryosinase," Chinese Traditinal and Herbal Drugs, vol. 4, pp. 168$169,1991$.

[103] Z. L. Liu, Y. F. Xu, C. X. Tu, and K. K. Wu, "Studies on the effect of ethanol extracts of 56 traditional Chinese drugs on the activity of tyrosinase," Journal of Dalian Medical University, vol. 22, no. 1, pp. 7-10, 2000.

[104] X. Y. Wang and J. X. Wang, "Synergistic effect of psoralen cooperated with substrates on tyrosinase activation," Natural Product Research and Development, vol. 19, no. 1, pp. 77-80, 2007.

[105] S. G. Deng, A. Q. Li, R. M. Ou, and Y. Q. Qu, "The anti-asthma effect of total-coumarines of fructus Psoraleae," Chinese Journal of Modern Applied Pharmacy, vol. 18, no. 6, pp. 439-440, 2001.

[106] W. X. Yu, W. Y. Li, H. Y. Li, L. Han, W. L. Chen, and S. G. Deng, "Effects of total coumarin on cGMP/cGMP of asthmatic rats," Research and Practice on Chinese Medicines, vol. 20, no. 5, pp. 27-29, 2006.

[107] J. X. Fu, "Measurement of MEFV in 66 cases of asthma in the convalescent stage and after treatment with Chinese herbs," Zhong Xi Yi Jie He Za Zhi, vol. 9, no. 11, pp. 658-659, 1989.

[108] A. Qamaruddin, N. Parveen, N. U. Khan, and K. C. Singhal, "Potential antifilarial activity of the leaves and seeds extracts of Psoralea corylifolia on cattle filarial parasite Setaria cervi," Journal of Ethnopharmacology, vol. 82, no. 1, pp. 23-28, 2002.

[109] W.-J. Tsai, W.-C. Hsin, and C.-C. Chen, "Antiplatelet flavonoids from seeds of Psoralea corylifolia," Journal of Natural Products, vol. 59, no. 7, pp. 671-672, 1996.

[110] R. P. Yang, Q. Y. Shou, and B. H. Wang, "Effect of Psoralea corylifolia L. on the proliferation and differentiation of osteoblast isolated from neonatal rat calvarium in vitro," China Practical Medical, vol. 2, no. 1, pp. 32-34, 2007.
[111] R. Q. Zhang, F. Q. Shi, S. Z. Pang, and S. F. Yu, "The effection of Psoralea corylifolia on isolated osteoclasts cells," Journal of Modern Stomatology, vol. 3, pp. 136-138, 1995.

[112] D. Wang, F. Li, and Z. Jiang, "Osteoblastic proliferation stimulating activity of Psoralea corylifolia extracts and two of its flavonoids," Planta Medica, vol. 67, no. 8, pp. 748-749, 2001.

[113] S.-H. Lim, T.-Y. Ha, S.-R. Kim, J. Ahn, H. J. Park, and S. Kim, "Ethanol extract of Psoralea corylifolia L. and its main constituent, bakuchiol, reduce bone loss in ovariectomised Sprague-Dawley rats," British Journal of Nutrition, vol. 101, no. 7, pp. 1031-1039, 2009.

[114] M.-H. Tsai, S. G. Huang, Y.-C. Hung, L. Bin, L.-T. Liao, and L.-W. Lin, "Psoralea corylifolia extract ameliorates experimental osteoporosis in ovariectomized rats," The American Journal of Chinese Medicine, vol. 35, no. 4, pp. 669-680, 2007.

[115] Y. Chen, L.-D. Kong, X. Xia, H.-F. Kung, and L. Zhang, "Behavioral and biochemical studies of total furocoumarins from seeds of Psoralea corylifolia in the forced swimming test in mice," Journal of Ethnopharmacology, vol. 96, no. 3, pp. 451-459, 2005.

[116] Y. Chen, H.-D. Wang, X. Xia, H.-F. Kung, Y. Pan, and L.D. Kong, "Behavioral and biochemical studies of total furocoumarins from seeds of Psoralea corylifolia in the chronic mild stress model of depression in mice," Phytomedicine, vol. 14, no. 7-8, pp. 523-529, 2007.

[117] Y. C. Chen, Y.-T. Cheung, L.-D. Kong et al., “Transcriptional regulation of corticotrophin releasing factor gene by furocoumarins isolated from seeds of Psoralea corylifolia," Life Sciences, vol. 82, no. 21-22, pp. 1117-1121, 2008.

[118] Q. Xu, Y. Pan, L.-T. Yi et al., "Antidepressant-like effects of psoralen isolated from the seeds of Psoralea corylifolia in the mouse forced swimming test," Biological \& Pharmaceutical Bulletin, vol. 31, no. 6, pp. 1109-1114, 2008.

[119] Y. B. Ji, Pharmachological Action and Application of Anticancer Traditional Chinese Medicine, Heilongjiang Science and Technology Press, 1990.

[120] Y. H. Choi, C. H. Yon, K. S. Hong et al., "In vitro BACE-1 inhibitory phenolic components from the seeds of Psoralea corylifolia," Planta Medica, vol. 74, no. 11, pp. 1405-1408, 2008.

[121] W. Q. Zhou, W. G. Zhang, and K. Y. Chen, "Advances in integrative medicine geriatrics," Journal of Shandong University of Traditional Chinese Medicine, vol. 7, no. 3, pp. 45-49, 1983.

[122] G. Z. Chai, G. H. Li, and Y. X. Guo, "The effection of Psoralea corylifolia, Agrjmonia pijosa on cysticercosis in vitro," Acta Chinese Medcie and Pharmacolocy, vol. 1991, no. 3, pp. 52-53, 1991.

[123] Y.-C. Kim, H. Oh, B. S. Kim et al., "In vitro protein tyrosine phosphatase $1 \mathrm{~B}$ inhibitory phenols from the seeds of Psoralea corylifolia," Planta Medica, vol. 71, no. 1, pp. 87-89, 2005.

[124] J. H. Choi, M.-C. Rho, S. W. Lee et al., "Bavachin and isobavachalcone, acyl-coenzyme A: cholesterol acyltransferase inhibitors from Psoralea corylifolia," Archives of Pharmacal Research, vol. 31, no. 11, pp. 1419-1423, 2008. 


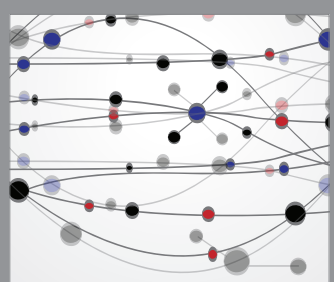

The Scientific World Journal
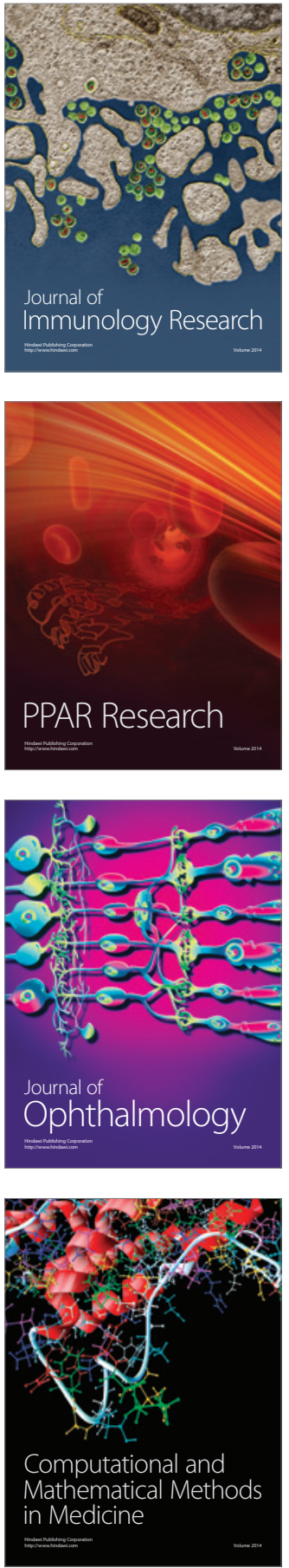

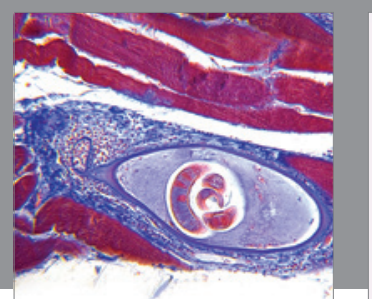

Gastroenterology Research and Practice

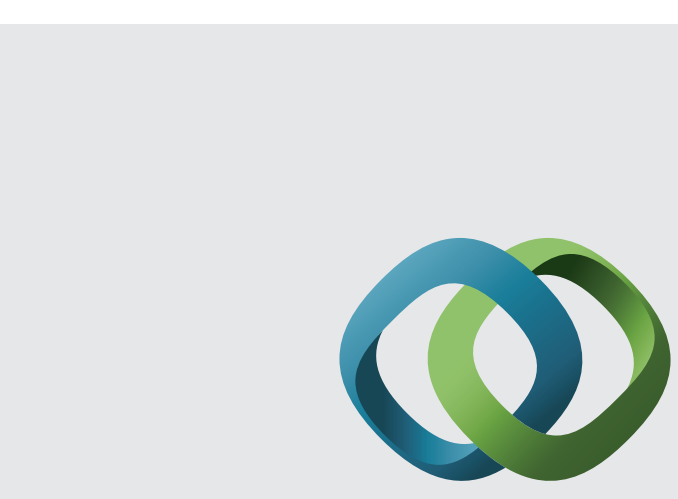

\section{Hindawi}

Submit your manuscripts at

http://www.hindawi.com
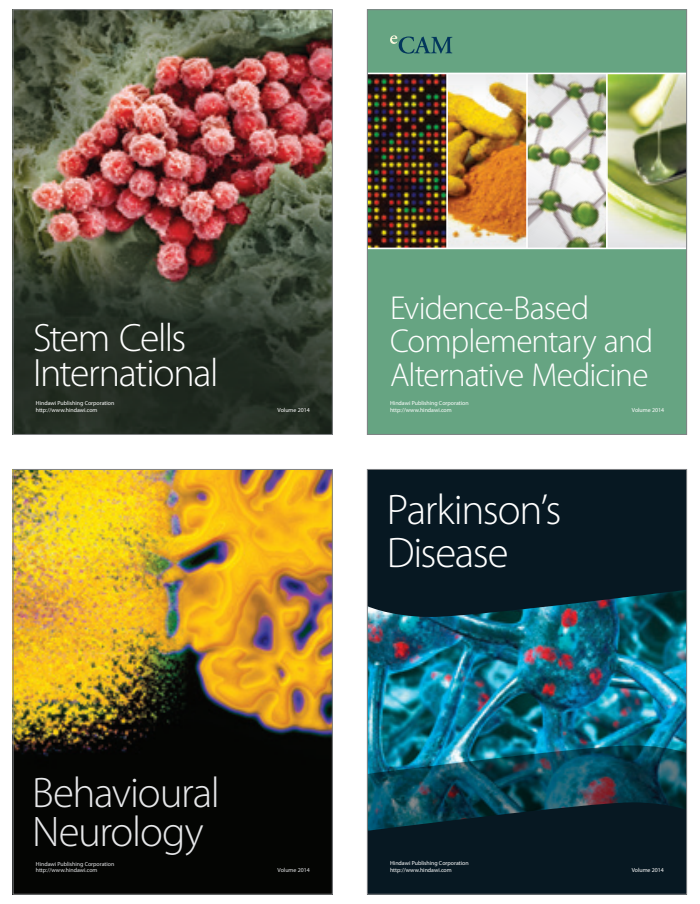
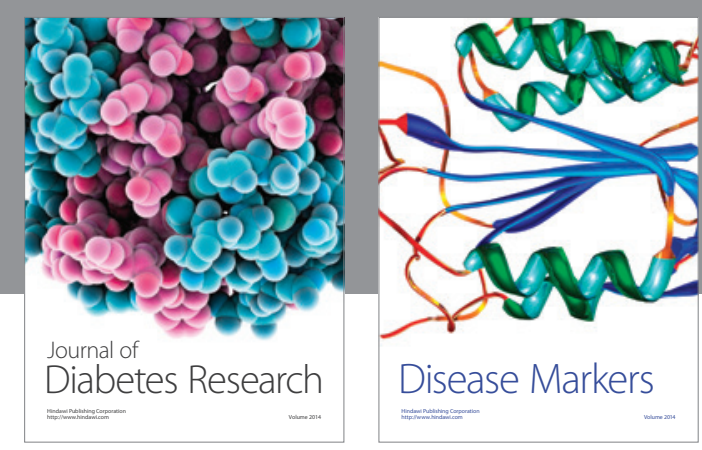

Disease Markers
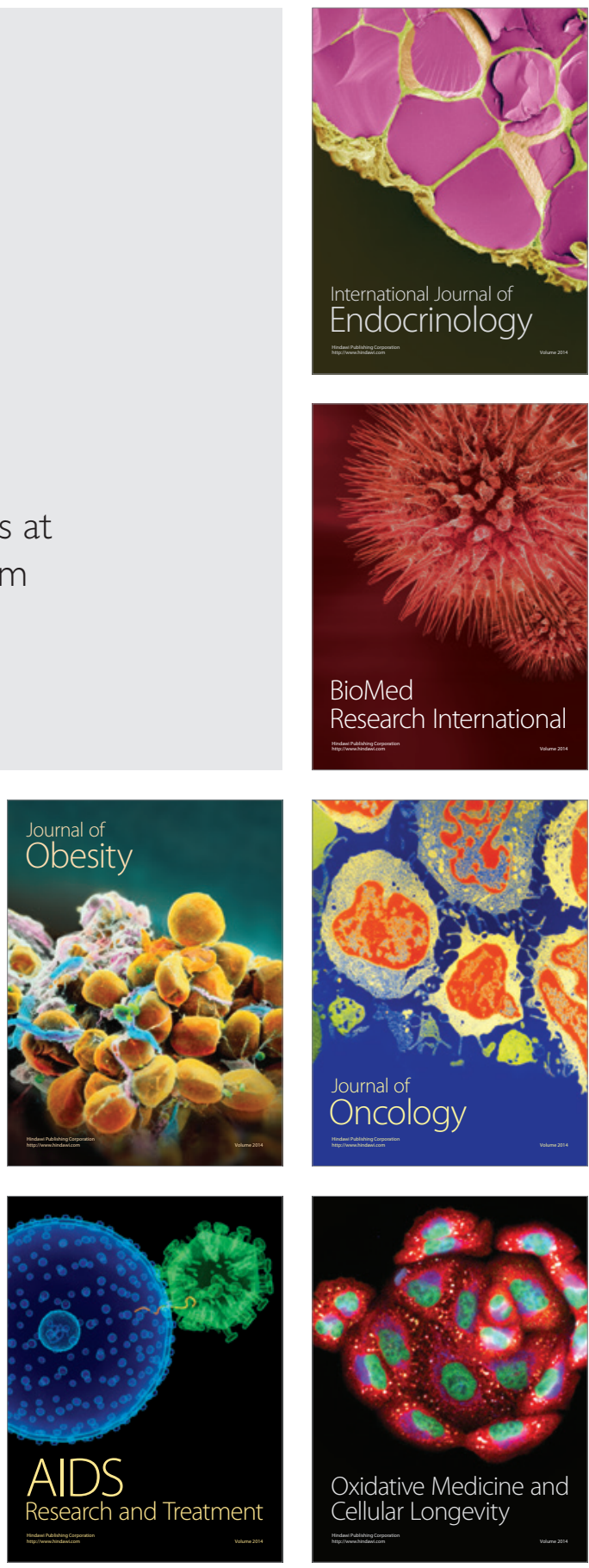